\title{
Faites-vous une idée de la démarche qualité des médecins
}

\section{Christoph Bosshard}

Dr méd., vice-président de la FMH, responsable du département Données, démographie et qualité

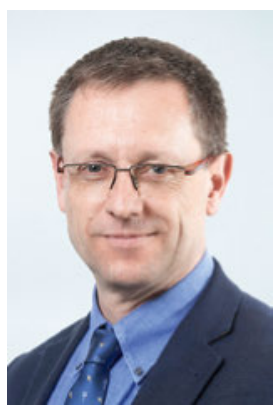

First they ignore you, then they laugh at you, then they fight you, then you win.

Mahatma Gandhi

Cette citation, attribuée à Gandhi, me vient régulièrement à l'esprit lorsque j'entends certaines déclarations à propos de la démarche qualité des médecins prononcées ces derniers temps en politique, formulées par l'administration et quelques assureurs. Le fait est que nos activités en lien avec la garantie et le développement de la qualité sont systématiquement scotomisées, et que cela exige donc encore davantage de communication de notre part. Puisqu'il est toujours bon de faire quelque chose de bien et d'en parler, nous, le corps médical, devons redoubler nos efforts de communication en ce sens.

\section{Sans savoir-faire et engagement des} membres et des organisations médicales, aucun projet novateur.

La FMH défend depuis des décennies le principe de transparence, comme le démontre la statistique médicale publiée depuis 1940 et l'enquête sur le revenu des médecins publiée depuis les années 70 jusqu'au veto de la Confédération en 2013. Après notre promesse tenue en 2012 avec la création de l'Académie suisse pour la qualité en médecine (ASQM), nous rappelons aujourd'hui le chemin parcouru au cours de ces cinq années dans un article-portrait publié dans ce numéro. Plusieurs des projets et des activités évoqués ont conservé leur caractère pionnier, ils se distinguent aussi par une innovation reconnue à l'international et sont portés avec force et engagement par les organisations concernées.
Le savoir-faire qui sous-tend ces initiatives et les travaux souvent réalisés bénévolement ne sont généralement pas perceptibles pour les personnes externes aux projets, ce qui confirme les défis de communication abordés ci-dessus. Si on veut référencer l'ensemble des efforts déployés en faveur de la qualité afin de pouvoir dégager une interprétation correcte du rapport coût-utilité, alors on ne peut pas se limiter aux seuls flux financiers. Une analyse est aussi nécessaire du côté de l'utilité incluant un regard global sur l'économie afin d'éviter de nouveaux incitatifs erronés.

Dans ce contexte, la question des charges administratives doit également être abordée. Le poids des tâches administratives auxquelles les médecins doivent faire face n'a cessé d'augmenter au cours des années les obligeant à passer davantage de temps à l'ordinateur ou à remplir des papiers au lieu de dédier ce temps à leur activité principale, le traitement des patients. N'est-ce pas là un des principaux problèmes en lien avec la qualité? Les démarches qualité ont donc aussi vocation de remettre les attentes et les besoins de nos patients au centre et non l'imagerie médicale, les résultats de laboratoire, les instructions de travail ou autre livre bleu, blanc ou noir. D'ailleurs un projet de l'ASQM existe aussi à ce sujet.

\section{Nos patients et leurs besoins doivent être} au cœur de toutes les démarches qualité.

Je vous invite donc à lire attentivement l'article sur l'ASQM à la page 630 et à consulter notre site Internet www.asqm.ch. Enfin, je remercie chaleureusement toutes nos organisations et nos membres qui ont travaillé sans relâche sur ces questions et qui y travaillent encore. Ce sont eux qui font l'ASQM. 